\title{
Revisitando as fases da abordagem centrada na pessoa
}

\author{
Revisiting the phases of the person centered approach
}

\author{
Virginia MOREIRA
}

\begin{abstract}
Resumo
Os interesses de Carl Rogers assumiram focos diferentes ao longo de seu pensamento, de maneira que seus comentadores dividiram sua teoria em distintas fases. Mais de 20 anos após sua morte, o que se observa atualmente é uma enorme diversidade de vertentes que se denominam Abordagem Centrada na Pessoa ou se dizem vinculadas a ela. Visando a contribuir para uma melhor compreensão do panorama atual da abordagem criada por Rogers, este artigo tem como objetivo revisitar as chamadas fases da Abordagem Centrada na Pessoa, propondo uma nova fase - a fase Pós-Rogeriana - consistente de vertentes atuais que, partindo de distintas fases daquela teoria, assumem distintos caminhos criando novas teorizações contemporâneas.
\end{abstract}

Unitermos: Carl Rogers. Psicologia humanistica. Terapia centrada no cliente.

\begin{abstract}
Carl Rogers'interests took on different focuses over the period of his thinking, such that his commentators divided his theory into different phases. More than 20 years after he passed away, what can be seen at the present time is a huge diversity of streams which call themselves Person Centered Approach or which are related to it. With the objective of contributing to a broader, present-day understanding of the approach created by Rogers, this article aims to revisit the so called phases of the Person Centered Approach and proposes a new phase-the Post-Rogerian Phase - which consists of present streams which, emerging from different phases of the Rogerian theory, assume different paths and create new, contemporary theorizations.
\end{abstract}

Uniterms: Carl Rogers. Humanistic psychology. Client centered terapy.

O pensamento de Carl Rogers sofreu uma evolução ao longo de sua carreira profissional, de tal maneira que a própria denominação de sua proposta teórica também foi se modificando. Em 1940, quando, segundo ele mesmo, nasce sua nova proposta teórica de psicoterapia, Rogers a nomeia de Psicoterapia Não-Diretiva ou Aconselhamento Não-Diretivo, tal como publicado em 1942 em seu livro Psicoterapia e consulta psicológica. Posteriormente passa a denominá-la Terapia Centrada na Cliente, Ensino Centrado no Aluno, Liderança Centra- da no Grupo e, por último, Abordagem Centrada na Pessoa, que, segundo ele, é a denominação mais adequada a sua teoria (C. Rogers, 1983). Essas mudanças na denominação de sua teoria devem-se aos diferentes interesses que Rogers foi assumindo como foco de seu trabalho ao longo da vida.

O ponto de partida foi seu trabalho clínico com crianças, publicado em 1939 no livro O tratamento clínico da criança problema, em que observa nelas o potencial positivo de desenvolvimento, que o levará a propor o

\footnotetext{
$\nabla \nabla \nabla \nabla$
}

1 Universidade de Fortaleza, Programa de Pós-Graduação em Psicologia, APHETO: Laboratório de Psicopatologia e Psicoterapia Humanista-Fenomenológica. Av. Washington Soares, 1321,60811-341, Fortaleza, CE, Brasil. E-mails: <virginiamoreira@unifor.br>; <virginia_moreira@hms.harvard.edu>. 
conceito de tendência atualizante, definido como uma tendência inerente, presente em todos os seres humanos, a desenvolver-se em uma direção positiva (C. Rogers, 1973; 1975; 1977a; 1977b; 1978a; 1978b; 1983). Esta passa a ser a ideia central ao longo de todo o seu pensamento, independentemente da denominação ou do foco de trabalho que venha a assumir.

O que ocorreu é que o primeiro Rogers, o psicoterapeuta, foi se ampliando no Rogers professor, facilitador de grupos, e o Rogers preocupado com processos sociais e questões como a paz mundial, fazendo com que ele buscasse ampliar sua teoria a esses vários campos. A denominação Abordagem Centrada na Pessoa (ACP) configura-se apenas em 1977, com a publicação de Sobre o poder pessoal. Para John Wood, um dos colaboradores mais próximos de Rogers, o período dos primeiros 30 anos - da Abordagem Centrada no Cliente - tinha como foco o desenvolvimento de um sistema de mudança na personalidade, concentrando-se no mundo subjetivo do indivíduo, enquanto os 30 anos seguintes - da Abordagem Centrada na Pessoa voltaram-se prioritariamente para interações sociais. Wood (1994) descreve a história da Abordagem Centrada na Pessoa através da seguinte evolução do foco teórico do pensamento rogeriano, com suas respectivas obras de referência: Atitudes do terapeuta (Psicoterapia e consulta psicológica, 1942), Métodos de terapia (Terapia Centrada no Cliente, 1951), Experiência ou processos internos (Tornar-se pessoa, 1961), Facilitação do aprendizado (Liberdade para aprender, 1969), Relacionamentos interpessoais (Grupos de encontro, 1970) e Processos sociais, formação e transformação de cultura (Sobre o poder pessoal, 1977 e Um jeito de ser, 1980).

A partir da evolução do foco teórico e metodológico ao longo do percurso intelectual de Carl Rogers, comentadores e colegas de trabalho (Hart \& Tomlinson, 1970; Huizinga, 1984; Puente, 1970; Wood, 1983) passaram a dividir seu pensamento em fases, ainda que ele mesmo nunca tenha se preocupado com isso. Segundo Holanda (1998), existe certa divergência entre as definições dessas fases: Puente, por exemplo, sugere: 1) A fase do Insight (1940-1945); 2) A fase da Congruência (1946-1957);3) A fase do Experiencing (1957-?). Já Huizinga refere-se a: 1) Terapia Não-Diretiva (que para Hart \& Tomlinson ocorre em 1940-1950); 2) Terapia Centrada no Cliente (1950-1957 segundo Hart\&Tomlinson), e 3) Terapia
Experiencial (1957-1964). Ou ainda, segundo Puente: 1) Início e meio da década de 1930, período em que o aconselhamento com crianças consistia na manipulação das condições do meio externo; 2) Fim da década de 1930 e início de 1940, quando Rogers desenvolveu o método não-diretivo, a partir de seu interesse na mudança terapêutica; 3) Fim da década de 1940, com a ênfase nas atitudes (aceitação positiva incondicional e compreensão empática); 4) Final da década de 1940 e início da década de 1950, com a inclusão da atitude de congruência.

A divisão em fases mais adotada tem sido a originalmente formulada por Hart \& Tomlinson (1970), citada por Cury (1987), colocada por Wood (1983) em seu esquema das tendências em meio século da Terapia Centrada na Pessoa e ampliada por Moreira (1990; 2001; 2007) e, posteriormente, por Holanda (1998): três fases referentes à psicoterapia; Fase Não-Diretiva (1940 - 1950), Fase Reflexiva (1950 - 1957), Fase Experiencial (1957 - 1970) e uma fase referente à ACP; Fase Coletiva (Moreira, 1990, 2001 e 2007) ou Inter-Humana (1970 - 1987).

\section{Fase não-diretiva (1940-1950)}

A primeira fase refere-se à Psicoterapia Não-Diretiva, durante o período em que Rogers estava na Universidade de Ohio, entre 1940 e 1950. A principal obra de referência desta fase é Counseling and psychotherapy, publicada em 1942 nos Estados Unidos, e em 1973 no Brasil, com o título Psicoterapia e consulta psicológica. A obra desenvolve as ideias que Rogers apresentara em sua palestra de 11 de dezembro de 1940, que ele define como o momento do nascimento de sua proposta inovadora de psicoterapia.

A Psicoterapia Não-Diretiva parte de conceitos que têm como base o impulso individual para o crescimento e para a saúde. Dá maior ênfase aos aspectos de sentimento do que aos intelectuais, enfatiza o presente do indivíduo em vez de seu passado, tem como maior foco de interesse o indivíduo e não o problema, e toma a própria relação terapêutica como uma experiência de crescimento. Essas ideias, por sua vez, eram provenientes de sua prática clínica com crianças no final da década de 1930.

Nessa fase, as atitudes do terapeuta privilegiam a técnica da permissividade, através de uma postura de 
neutralidade em que o profissional deveria intervir o mínimo possível (Holanda, 1998). Segundo Wood (1994), o psicoterapeuta (ou "conselheiro", termo mais utilizado nessa etapa) renuncia ao papel de especialista, tornando mais pessoais as relações com o cliente e conduzindo a sessão a partir da orientação ditada por este último (denominado "paciente" em Psicoterapia e consulta psicológica). Ou seja, é o cliente quem conduz o processo, e não o psicoterapeuta, que, intervindo minimamente, pretende não dirigir a sessão, deixando que o primeiro o faça.

A ideia de não-diretividade teve severos críticos, principalmente na França (Moreira, 1990; 2001; 2007), ocasionando mitos e mal-entendidos de laissez-faire sobre a atuação do terapeuta que não fala na sessão, quando, na verdade, o esforço de Rogers era no sentido de desconstruir a figura de autoridade do psicoterapeuta (Holanda, 1998). Ainda que o próprio Rogers tenha deixado de usar esta denominação desde a década de 50, por entender que não era uma denominação adequada, ainda hoje, eventualmente escuta-se falar em "não-diretividade" quando se fala no pensamento de Carl Rogers, possivelmente pela popularidade do termo, que acabou contribuindo para imagens frequentemente negativas dessa abordagem (Moreira, 2001; 2007).

\section{Fase reflexiva (1950-1957)}

Nesta fase, entre 1950 e 1957, o reflexo de sentimentos é muito utilizado, daí sua denominação. É a fase da Terapia Centrada no Cliente, sendo a função do terapeuta promover o desenvolvimento do cliente em uma atmosfera desprovida de ameaça, isto é, sob condições facilitadoras (Gobbi \& Missel, 1998). Aqui a noção de "não-direção" é substituída pela de "centramento no cliente", sugerindo um papel mais ativo por parte do terapeuta e transformando o cliente no foco maior de sua atenção (Cury, 1987), enquanto na fase anterior a ênfase recaía sobre a não-direção do processo, com um psicoterapeuta mais passivo.

Essa fase corresponde ao período em que Rogers esteve em Chicago e tem como obra de referência o livro Psicoterapia Centrada no Cliente, de 1951 (Holanda, 1998; Gobbi \& Missel, 1998), no qual a renúncia a teorias rígidas é parte importante do pensamento rogeriano, e a psicoterapia pode ser entendida como um grupo de duas pessoas (Wood, 1983). É desenvolvida a teoria das atitudes facilitadoras, segundo a qual o psicoterapeuta deve apresentar três condições para que ocorra o crescimento do cliente: empatia, aceitação positiva incondicional e congruência. Através da empatia, o psicoterapeuta busca perceber e compreender o mundo do cliente na perspectiva dele. A aceitação positiva incondicional consiste no respeito incondicional, por parte do psicoterapeuta, à individualidade do cliente. A congruência, ou autenticidade, é descrita como o grau de correspondência entre o que o terapeuta experiência e o que comunica ao cliente, sendo ele mesmo na relação terapeuta-cliente. Em um de seus artigos seminais, "As condições necessárias e suficientes para a mudança terapêutica de personalidade", publicado em 1957, Rogers (1994) desenvolve a teoria das atitudes facilitadoras do processo terapêutico, assim resumindo o que, para ele, são as condições necessárias e suficientes para que ocorra uma mudança construtiva de personalidade:

1) Que duas pessoas estejam em contato psicológico; 2) Que a primeira, a quem chamaremos cliente, esteja num estado de incongruência, estando vulnerável ou ansiosa; 3) Que a segunda pessoa, a quem chamaremos de terapeuta, esteja congruente ou integrada na relação; 4) Que o terapeuta experiencie consideração positiva incondicional pelo cliente; 5) Que o terapeuta experiencie uma compreensão empática do esquema de referência interno do cliente e se esforce por comunicar esta experiência ao cliente; 6) Que a comunicação ao cliente da compreensão empática do terapeuta e da consideração positiva incondicional seja efetivada, pelo menos num grau mínimo (p.157).

Com base no desenvolvimento teórico das atitudes facilitadoras por parte do psicoterapeuta, Rogers enfatiza o abandono do interesse diagnóstico, sempre priorizando a capacidade de desenvolvimento inerente à pessoa.

\section{Fase experiencial (1957-1970)}

É a fase da Terapia Experiencial, ou período de Wisconsin, compreendida entre 1957 e 1970, e iniciada com o livro On becoming a person, de 1961, publicado no Brasil em 1976 sob o título Tornar-sepessoa, sua obra de referência. Foi classificada como experiencial devido 
à mudança da conduta rogeriana que, influenciada pelo conceito de experienciação, de Eugene Gendlin, passou a focalizar a experiência vivida do cliente, do psicoterapeuta e entre ambos (Cury, 1987; 1988). A partir dessa influência, que priorizou o foco do processo terapêutico na experiência, a intervenção do profissional passa a ter lugar no espaço da relação intersubjetiva terapeuta-cliente, ainda que Rogers, em sua prática clínica, em muitos momentos voltasse a colocar como centro o cliente, em lugar da experiência intersubjetiva (Moreira, 2001;2007).

Nessa fase, o objetivo da psicoterapia é ajudar o cliente a usar plenamente sua experiência no sentido de promover uma maior congruência do self e do desenvolvimento relacional. Ou seja, a ênfase recai sobre a vida inter e intrapessoal, e a relação terapêutica passa a adquirir significado enquanto encontro existencial (Gobbi \& Missel, 1998; Holanda, 1998). Aqui é enfatizada a autenticidade do terapeuta enquanto atitude facilitadora. O psicoterapeuta deve confiar em seus próprios sentimentos, sendo congruente com a própria experiência; ou seja, a sua experiência passa a ser entendida como parte da relação terapeuta-cliente. É nesse sentido que a relação deixa de ser entendida como centrada no cliente, para ser compreendida como bicentrada, visto que consiste em um esforço para explorar dois mundos fenomênicos, fazendo-os interatuar em benefício do cliente através da criação de novos significados a partir do espaço experienciado por ambos. O psicoterapeuta utiliza-se de seus sentimentos como movimentos dirigidos ao cliente, ocorrendo um diálogo íntimo e intersubjetivo entre ambos (Cury, 1987; 1988; Moreira, 2001; 2007). Nessa fase, Rogers trabalha mais intensamente com colegas colaboradores, deles aceitando sugestões e críticas. Entre eles encontra-se Gendlin, que, segundo Spiegelberg (1972), influência teoricamente Rogers no sentido da passagem do positivismo lógico para uma orientação existencialista, possibilitando a reinterpretação do termo "experiência".

Em sua análise da evolução das formulações sobre a relação terapeuta-cliente na Psicoterapia Centrada na Pessoa, Cury (1987) assinala a contribuição especial de Gendlin, entre outros colegas de Rogers, para a descrição dessa fase. Gendlin (1970a,b) criou uma ramificação importante da Abordagem Centrada na

540 Pessoa, com uma proposta psicoterapêutica baseada em uma teoria experiencial, que priorizava a vivência da relação terapeuta-cliente, estando mais voltada para a experiência dessa relação do que para o conteúdo verbal em si mesmo. Essa experiência é considerada responsável pelo processo de mudança do cliente (Moreira, 2001; 2007).

No entanto, o próprio Rogers não chegou à teorização do processo psicoterapêutico entendido como um fluxo experiencial. A fase experiencial de seu pensamento passa a ser descrita a partir da ênfase no conceito de autenticidade do terapeuta, na experiência imediata da relação com o cliente (Cury, 1987) - e aqui vale lembrar a importância da definição do conceito de autenticidade como estar presente na experiência da relação terapêutica. Esse conceito é frequentemente confundido, levando a pensar que ser autêntico significa falar de si. Nas palavras de Rogers (1976):

Descobriu-se que a transformação pessoal era facilitada quando o psicoterapeuta é aquilo que é, quando as suas relações com o paciente são autênticas e sem máscara nem fachada, exprimindo abertamente os sentimentos e as atitudes que nesse momento Ihe ocorrem. Escolhemos o termo "congruência" para tentar descrever esta condição. Com este termo procura-se significar que os sentimentos experimentados pelo terapeuta lhe são disponíveis, disponíveis à sua consciência, e que ele é capaz de vivê-los, de ser esses sentimentos e estas atitudes, que é capaz de comunicá-los se surgir uma oportunidade disso (p.63).

Essa é a fase em que a prática clínica rogeriana mais se aproxima das abordagens de tradição fenomenológica, com a passagem da focalização na pessoa do cliente para a focalização na experiência intersubjetiva. É o que mostra pesquisa (Moreira, 2001; 2007) que analisou sessões de psicoterapia de Rogers em diferentes fases, apontando, no entanto, que a direção fenomenológica, que enfatiza a experiência intersubjetiva na relação psicoterapêutica, acaba se perdendo na fase posterior do autor estudado. A pesquisa conclui que:

a concepção de pessoa como centro, impede Rogers de realizar uma psicoterapia fenomenológica. Mais do que isso, o centramento na pessoa direciona, restringe, e pela mesma razão, empobrece o processo terapêutico, tal como se observou no exemplo mais ortodoxo - a entrevista de Bryan - e na entrevista aparentemente menos centrada - de Jan. A análise 
da prática clínica rogeriana mostra que esta caminha em direção à fenomenologia; da pessoa como centro para a experiência, o que se pôde ver na entrevista com Glória, durante a fase experiencial. Entretanto, para que o modelo de psicoterapia que nos deixou Carl Rogers possa assumir todo seu potencial de contribuição fenomenológica, é necessário que deixe, definitivamente, a busca de um suposto homem interno - a pessoa - voltando-se para uma terapia do fenômeno emergente que, como demonstra o mesmo Rogers, já existe potencialmente embrionária em sua proposta (Moreira, 2007, p.218).

Esta ideia, no entanto, não foi assumida por Rogers, que continuou a falar de abordagem centrada na pessoa até o fim da vida.

\section{Fase coletiva ou inter-humana (1970-1987)}

Moreira (1990;2001; 2007) propõe a inclusão dessa quarta fase, às anteriormente propostas por Hart \& Tomlinson (1970), Wood (1983) e Cury (1987), observando que nos últimos 15 anos de sua vida Rogers voltou seu interesse para questões mais amplas, concernentes às atividades de grupo e à relação humana coletiva, abandonando definitivamente a atividade de terapia individual no consultório e assumindo em seu trabalho a definição de abordagem, em vez de psicoterapia (Wood, 1994; 2008). A necessidade de descrever o que acontece nos últimos anos do trabalho de Rogers é corroborada por Wood (1983) e retomada por Holanda (1998), que, utilizando-se de uma linguagem buberiana, em lugar de "fase coletiva", tal como proposta originalmente por Moreira (1990), propõe a denominação"fase inter-humana", por considerar que "coletiva privilegia demasiado uma outra dimensão da existência humana, a social, representada pelo grupo onde temos a realização desse coletivo, mas que, em geral, suprime o elemento pessoal individual, justamente o elemento mais importante" (p.110).

Holanda (1998) justifica a denominação "inter-humana", argumentando que esta é uma fase de transcendência de valores e de ideias, em que Rogers trabalha com conceitos que coexistem em outras áreas da ciência, tais como a física, a química ou a biologia, expressando sua preocupação com o futuro do homem e do mundo. "Seria uma fase mais mística, holística em seu sentido amplo, em que Rogers se voltaria para a consideração de uma relação mais transcendental, ou para a transcendência da existência humana"(p.110).Tal ponto de vista pode ser ilustrado através da seguinte afirmação de C. Rogers (1983):

Defendo a hipótese de que existe uma tendência direcional formativa no universo, que pode ser rastreada e observada no espaço estelar, nos cristais nos microrganismos, na vida orgânica mais complexa e nos seres humanos ... Na espécie humana, essa tendência se expressa quando o indivíduo progride de seu início unicelular para um funcionamento orgânico complexo, para um modo de conhecer e de sentir abaixo do nível da consciência, para um conhecimento consciente do organismo e do mundo externo, para uma consciência transcendente da harmonia e da unidade do sistema cósmico, no qual se inclui a espécie humana (p.50).

Faz sentido, portanto, a leitura de Holanda (1998), com relação a esse período. É o momento em que Rogers assume a denominação de Abordagem Centrada na Pessoa, a partir de 1976, formalizada na publicação de On personal Power, de 1977, editado em português sob o título Sobre o poder pessoal. Poder-se-ia nomear como obra de referência dessa fase A way of being, publicado em 1980 nos Estados Unidos, e em 1983 publicado parcialmente no Brasil, sob o título Um jeito de ser. Nesta obra, C. Rogers (1983) afirma:"O que entendo por abordagem centrada na pessoa?... Sorrio quando penso nos diversos rótulos que dei a esse tema no decorrer da minha carreira - aconselhamento não-diretivo, terapia centrada no cliente, ensino centrado no aluno, liderança centrada no grupo. Como os campos de aplicação cresceram em número e variedade, o rótulo 'abordagem centrada na pessoa' parece ser o mais adequado" (p. 38).

\section{Quinta fase pós-rogeriana ou neorrogeriana}

Revisitar o pensamento de Carl Rogers na contemporaneidade, mais de 20 anos após sua morte, leva-nos a retomar questões importantes: "Sabe-se que a Psicoterapia Centrada na Pessoa tem sido utilizada por outros profissionais além de Rogers e continua existindo. Como está sendo praticada e pensada por esses profissionais?" (Moreira, 2007, p.218).

Nos últimos 20 anos, desde a morte de Carl Rogers em 1987, a Abordagem Centrada na Pessoa tem se desenvolvido através uma grande diversidade de 
vertentes, em distintos lugares do mundo. Na Inglaterra, Sanders (2007) refere-se às escolas de terapia relacionadas à Abordagem Centrada na Pessoa: clássica, focalização, experiencial, existencial, integrativa. Segrera (2002) descreve alguns dos desenvolvimentos atuais da Abordagem Centrada na Pessoa em vários outros lugares do mundo: 1) a versão clássica, atualmente desenvolvida pelo Center for Studies of the Person, onde Rogers passou a última fase de sua vida; 2) a linha experiencial fundada por Gendlin (1988; 1990), com ênfase na experienciação e focalização, na University of Chicago; 3) a linha experiencial processual, representada por Laura Rice, no Canadá e Robert Elliot, nos Estados Unidos (Greenberg, Rice \& Elliot, 1993 ; Rice \& Greenberg, 1990), com interesse principal no estudo detalhado dos elementos do processo; 4) a linha existencial-fenomenológica, embasada na fenomenologia existencial, desenvolvida principalmente por autores brasileiros e, segundo Segrera (2002), representada por Moreira (2001) - a quem podem-se acrescentar outros representantes no Brasil, como Advíncula (1991), Amatuzzi (1989), Belém (2004), Boris (1987; 1990), Cury (1987; 1988; 1993), Fonseca (1988; 1998) e Holanda (1998), entre outros; 5) a linha transcendental, que abarca interesses espirituais, religiosos e transpessoais, trabalhada por autores como Curran (1952) nos Estados Unidos, Saint-Arnaud (1967) no Canadá, Thorne (1993) na Inglaterra, Schmid (1995) na Áustria, González (1995) no México, Boainain Jr. (1999) no Brasil; 6) a linha expressiva, que integra elementos de arte e movimento corporal, estabelecendo pontes com a gestalt-terapia e o psicodrama, sendo representada principalmente pela filha de Carl Rogers, N. Rogers (1993); 7) a linha analítica, com interesse na relação entre a psicologia do si mesmo de Heinz Kohut e outros elementos analíticos, representada por Kahn (1985); 8) a linha comportamental-operacional, com ênfase no desenvolvimento de habilidades, representada por Tausch (1990), na Alemanha e Ernest Meadows (Meadows \& Stillwell, 1998), na Califórnia; Moreira (2008; 2009) acrescenta um nono desenvolvimento contemporâneo: 9) a linha do curriculum centrado na pessoa, realizado na área de educação, no Chile, representado por Eric Troncoso e Ana Repetto (Moreno, Troncoso \& Videla, 1999; Troncoso \& Repetto, 1997). Recentemente é possível, ainda, observar outros desenvolvimentos que podem vir a constituir novas linhas.
É interessante observar que essas diferentes vertentes pós-rogerianas partem de fases diferentes do pensamento de Carl Rogers. Por exemplo, a linha existencial- fenomenológica - ou humanista-fenomenológica (Moreira, 2008; 2009) - parte da fase experiencial da psicoterapia de Carl Rogers (1957-1970), acentuando seu caráter fenomenológico através de contribuições da tradição da Psicopatologia Fenomenológica e da Análise Existencial. Utiliza-se do potencial eminentemente compreensivo dessa abordagem para o desenvolvimento teórico e metodológico de uma clínica ampliada crítica, ou mundana, que tem como fundamento as filosofias existenciais de autores como Heidegger, Merleau-Ponty e Buber, entre outros. Já a linha transcendental toma como base especialmente os escritos do último Rogers, da fase inter-humana (1970-1987), quando ele deixa de ser psicoterapeuta e se volta para questões mais transcendentais do ser humano.

Esse fato tem sérias implicações tanto metodológicas como epistemológicas, pois a fundamentação que será adotada por cada uma das linhas variará segundo seu desenvolvimento depois de Rogers. Nesse sentido é que elas passam a ser neorrogerianas, assumindo identidade própria. Assim, ainda tomando o mesmo exemplo, a linha humanista-fenomenológica terá como base a ideia de homem mundano e do trabalho clínico voltado para a compreensão do Lebenswelt (mundo vivido), enquanto a linha transcendental terá uma fundamentação espiritual, com trabalho clínico norteado por valores religiosos ligados aos aspectos transpessoais do ser humano.

\section{Considerações Finais}

Revisitar o pensamento de Carl Rogers na contemporaneidade traz à tona a necessidade de uma atualização das fases da Abordagem Centrada na Pessoa, que podem então ser assim descritas: três fases referentes à psicoterapia; Fase Não-Diretiva (1940 - 1950), Fase Reflexiva (1950 - 1957), Fase Experiencial (1957 - 1970), e duas fase referentes à ACP; Fase Inter-Humana (1970-1987) e Fase Pós-Rogeriana ou Neorrogeriana (1987-atual)

Uma análise mais detalhada dos vários desdobramentos atuais do pensamento de Rogers em tantas 
vertentes diversas, com diferentes bases epistemológicas, mostra que diferentes vertentes atuais partem de fases distintas do pensamento rogeriano. De qual Rogers está se falando? Do psicoterapeuta? Do professor? Do homem mais preocupado com questões transcendentais? Do que tem como foco uma tendência atualizante ou do que se refere a uma tendência formativa do universo?

Na medida em que pensar a Abordagem Centrada na Pessoa na atualidade inclui tamanha diversidade, é importante não perder de vista as distintas fases desse pensamento, no sentido de compreender as aproximações e as divergências entre as várias vertentes que constituem a fase Pós-Rogeriana ou Neorrogeriana.

Observa-se que são várias as vertentes atuais, provenientes direta ou indiretamente do pensamento de Carl Rogers. Chama atenção a diversidade dessas vertentes, todas elas configurações contemporâneas da Abordagem Centrada na Pessoa ou, pelo menos, originadas dela. Não se trata mais do pensamento de Rogers puro, mas de novas teorizações variadas, que partem dele. Na verdade, o próprio Rogers não se pretendeu purista nem cristalizado em nenhuma teoria, nem mesmo a dele, afirmando durante sua última visita ao Brasil, em 1985, que não era rogeriano Nesse sentido, é importante não apenas não ignorar os significativos desdobramentos em andamento nos últimos 20 anos após sua morte, como estabelecer um diálogo entre as diferenças que preserve a proposta original de Carl Rogers em seu caráter humanista, de respeito pelo ser humano e suas potencialidades. Seu pensamento continua vivo em cada uma das vertentes atuais, mesmo que seus distintos desenvolvimentos - originados de fases diversas do pensamento rogeriano e, portanto, passando a assumir diferentes caminhos epistemológicos na continuidade de sua construção teórica - as tornem tantas vezes tão diferenciadas entre si.

O potencial fenomenológico da teoria de Carl Rogers é, sem dúvida, um eixo denso, que merece especial atenção no que se refere à continuidade de sua construção teórica, na direção de uma clínica humanista-fenomenológica crítica e ampliada, que priorize o acolhimento da alteridade. Mas esse não é o único caminho. Ignorar a pluralidade e as diferenças seria perder-se da proposta original do próprio Rogers.

\section{Referências}

Advíncula, I. (1991). Tendência atualizante e vontade de potência: um paralelo entre Rogers e Nietzsche. Psicologia: Terapia e Pesquisa, 7 (2), 201-214.

Amatuzzi, M. (1989). O resgate da fala autêntica: filosofia da psicoterapia e da educação. Campinas: Papirus.

Belém, D. (2004). Abordagem centrada na pessoa: um olhar contemporâneo. Dissertação de mestrado não-publicada, Universidade Católica de Pernambuco, Recife.

Boanain Jr., E. (1999). Tornar-se transpessoal: transcendênciae espiritualidade na obra de Carl Rogers. São Paulo: Summus.

Boris, G. (1987). Uma reflexão acerca da consistência teórica das psicoterapias humanistas. Revista de Psicologia, 5 (1), 69-75.

Boris, G. (1990). Abordagem centrada na pessoa e GestaltTerapia. Revista de Humanidades, 7 (5), 111-124.

Curran, C. (1952). Counseling in catholic life and education. New York: MacMillan.

Cury, V. (1987). Psicoterapia centrada na pessoa: evolução das formulações sobre a relação terapeuta-cliente. Dissertação de mestrado não-publicada, Universidade de São Paulo.

Cury, V.E. (1988). ACP: encruzilhada de perspectivas. Boletim da Abordagem Centrada na Pessoa, (Edição Especial).

Cury, V.E. (1993). A Abordagem centrada na pessoa: um estudo sobre as implicações dos trabalhos com grupos intensivos para a terapia centrada no cliente. Tese de doutorado não-publicada, Universidade Estadual de Campinas.

Fonseca, A. (1988). Grupo fugacidade, ritmo e forma: processo de grupo e facilitação na psicologia humanista. São Paulo: Ágora.

Fonseca, A. (1998). Trabalhando o legado de Rogers: sobre os fundamentos fenomenológicos existenciais. Maceió: Pendang.

Gendlin, E. (1970a). A theory of personality change. In J. Hart \& T. Tomlinson (Eds.), New directions in client-centered therapy. Boston: Houghton Mifflin.

Gendlin, E. (1970b) Existencialism and experimental psychotherapy. In J. Hart \& T. Tomlinson (Eds.), New directions in client-centered therapy. Boston: Houghton Mifflin.

Gendlin, E. (1988). Focusing: proceso y técnica del enfoque corporal. Bilbao: Mensajero.

Gendlin, E. (1990). The small steps of the therapy process: how they come and how to help to come. In G. Lietaer, J. Rombauts \& R. Van Balen. Client-centered and experiential psychotherapy in the nineties (pp.205-224). Leuven: Leuven University Press.

Gobbi, S., \& Missel, S. (1998). Abordagem centrada na pessoa: vocabulário e noções básicas. Tubarão: Universitária.

González, A. M. (1995). Dela sombra a la luz: desarrollo humano transpersonal. México: Editorial Jus \& Universidad Iberoamericana. 
Greenberg, L., Rice, L., \& Elliot, R. (1993). Facilitating emotional change: the moment by moment process. New York: Guilford.

Hart, J. T., \& Tomlinson, T. M. (1970). New directions in clientcentered therapy. Boston: Houghton Miffin.

Holanda, A. (1998). Diálogo e psicoterapia: correlações entre Carl Rogers e Martin Buber. São Paulo: Lemos.

Huizinga, J. (1984) Developments in life and work of Carl Ramsom Rogers. In A. Segrera (Ed.), Proceedings of the First International Forum on the Person Centered Approach. Mexico: Universidad Iberoamericana.

Khan, E. (1985). Heinz Kohut and Carl Rogers: toward a construtctive collaboration. Psychotherapy, 26 (4), 555-563.

Meadows, E., \& Stillwell, W. (1998). Voicing congruence [interview]. In W. Stillwell (Ed.), Questing voices: interviews with person-centered practioners (pp.5-52). La Jolla: Center for Studies of the Person.

Moreira, V. (1990). Para além da pessoa: um estudo crítico da psicoterapia de Carl Rogers. Tese de doutorado nãopublicada, Pontificia Universidade Católica de São Paulo.

Moreira, V. (2001). Más allá de la persona: hacia uma psicoterapia fenomenológica mundana. Santiago: Editorial Universitario Universidad de Santiago de Chile.

Moreira, V. (2007). De Carl Rogers a Merleau-Ponty: a pessoa mundana em psicoterapia. São Paulo: Annablume.

Moreira, V. (2008). Psicología humanista fenomenológica. In A. Kaulino \& A. Stecher (Eds.), Cartografía de la psicología contemporánea (pp.167-191). Santiago de Chile: LOM-Serie Universitaria.

Moreira, V. (2009). Clínica humanistafenomenológica:estudos em psicoterapia e psicopatologia crítica. São Paulo: Annablume.

Moreno, A. Troncoso, R., \& Videla, A. (1999). Del profesor tradicional hacia um facilitadorcentrado en la persona. Tesis non-publicación, Universidad Academia de Humanismo Cristiano, Chile.

Puente, M. (1970). Carl Rogers: de la psychothérapie à I'enseignement. Paris: EPI Editeurs.

Rice, L, \& Greenberg, L. (1990). Fundamental dimensions in experiential therapy: new directions in research. In G. Lietaer, J. Rombauts \& R. Van Balen. Client-centered and experiential psychotherapy in the nineties (pp.397-414). Leuven: Leuven University Press.

Rogers, C. (1973). Psicoterapia e consulta psicológica. São Paulo: Martins Fontes.

Rogers, C. (1975). A terapia centrada no cliente. São Paulo: Martins Fontes.

Rogers, C. (1976). Tornar-sepessoa. São Paulo: Martins Fontes.

Rogers C. (1977a). Definições das noções teóricas. In C. Rogers \& M. Kinget (Eds.), Psicoterapia e relações humanas (Vol 1, pp.157-179). Belo Horizonte: Interlivros.
Rogers C. (1977b). O funcionamento ótimo da personalidade (Vol. 1, pp.255-272). In C. Rogers \& M. Kinget. (Eds.), Psicoterapia e relações humanas. Belo Horizonte: Interlivros.

Rogers, C. (1978a). O tratamento clínico da criança problema. São Paulo: Matins Fontes.

Rogers, C. (1978b). Sobre o poder pessoal. São Paulo: Martins Fontes.

Rogers, C. (1983). Um jeito de ser. São Paulo: E.P.U.

Rogers, C. (1994). As condições necessárias e suficientes para a mudança terapêutica da personalidade. In J. Wood et al. (Orgs.), Abordagem centrada na pessoa (pp.155-177). Vitória: Editora Fundação Ceciliano Abel de Almeida.

Rogers, N. (1993). The creative connection: expressive arts as healing. Palo Alto: Science \& Behavior.

Sanders, P. (Ed.) (2007). The tribes of the person-centered nation. Trowbridge: Cromwell.

Saint Arnaud, Y. (1967). La consultation pastorale d'orientation rogérienne. Bruxelles: Desclée de Brower.

Schmid, P. (1995). Personale begegnun: der personzentrierte ansatz in psychotherapie: beratung, gruppenarbeit und seelsorge $\left(2^{\text {nd }}\right.$ ed.) Würzburg, Bayern: Echter.

Segrera, A. (2002). El enfoque centrado en la persona: reflexiones en el centenario de su fundador Carl Rogers. Miscelánea Comillas, 60 (117), 399-419.

Spiegelberg, H. (1972). Phenomenology in psychology and psychiatry. Evanston: Northwester University Press.

Tausch, R. (1990). The supplementation of client-centered communication therapy with other valid therapeutic methods (pp.447-456). In In G. Lietaer, J. Rombauts \& R. Van Balen. Client-centered and experiential psychotherapy in the nineties. Leuven: Leuven University Press.

Thorne, B. (1993). Person-centered counselling: therapeutic and spiritual dimensions. London: Whurr.

Troncoso, E, \& Repetto, A. (1997). Curriculum centrado en la persona: el milagro de creer en las capacidades del niño. Santiago: The Angels School.

Wood, J. K. (1983). Terapia de Grupo Centrada na Pessoa. In C. Rogers, J. K. Wood, M. M. O’Hara \& A. Fonseca. Em busca de vida: da terapia centrada no cliente à abordagem centrada na pessoa (pp.45-90). São Paulo: Summus,

Wood, J. K. (1994). Prólogo. In J. K. Wood et al. (Orgs.), Abordagem centrada na pessoa (pp.I-XIV). Vitória: Editora Fundação Ceciliano Abel de Almeida.

Wood, J. K. (2008). Carl Rogers' person-centered approach: toward an understanding of its implications. Herforshire: PCCS Books.

Recebido em: 31/8/2009

Versão final reapresentada em: 12/4/2010

Aprovado em: 20/5/2010 